IN THE MID-2000s Harry Sokol, a gastroenterologist at Saint Antoine Hospital in Paris, was surprised by what he found when he ran some laboratory tests on tissue samples from his patients with Crohn's disease, a chronic inflammatory disorder of the gut. The exact cause of inflammatory bowel disease remains a mystery. Some have argued that it results from a hidden infection; others suspect a proliferation of certain bacteria among the trillions of microbes that inhabit the human gut. But when Sokol did a comparative DNA analysis of diseased sections of intestine surgically removed from the patients, he observed a relative depletion of just one common bacterium, Faecalibacterium prausnitzii. Rather than "bad" microbes prompting disease, he wondered, could a single "good" microbe prevent disease?

Sokol transferred the bacterium to mice and found it protected them against experimentally induced intestinal inflammation. And when he subsequently mixed $F$ prausnitzii with human immune cells in a test tube, he noted a strong anti-inflammatory response. Sokol seemed to have identified a powerfully anti-inflammatory member of the human microbiota.

Each of us harbors a teeming ecosystem of microbes that outnumbers the total number of cells in the human body by a factor of 10 to one and whose collective genome is at least 150 times larger than our own. In 2012 the National Institutes of Health completed the first phase of the Human Microbiome Project, a multimillion-dollar effort to catalogue and understand the microbes that inhabit our bodies. The microbiome varies dramatically from one individual to the next and can

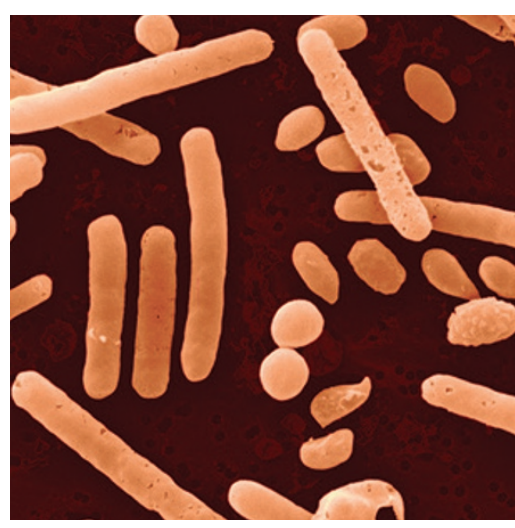

UNRULY NAMESAKE: Clostridium difficile, a bacterial scourge in hospitals, is a distant relative of benign "clostridial cluster" microbes that seem to play a key role in gut health. clostridial group. Dubbed "clostridial clusters," these microbes are distantly related to Clostridium difficile, a scourge of hospitals and an all too frequent cause of death by diarrhea. But where $C$. difficile prompts endless inflammation, bleeding and potentially catastrophic loss of fluids, the clostridial clusters do just the opposite - they keep the gut barrier tight and healthy, and they soothe the immune system. Scientists are now exploring whether these microbes can be used to treat a bevy of the autoimmune, allergic and inflammatory disorders that have increased in recent decades, including Crohn's and maybe even obesity.

F. prausnitzii was one of the first clostridial microbes to be identified. In Sokol's patients those with higher counts of $F$. prausnitzii consistently fared best six months after surgery. After he published his initial findings in 2008, scientists in India and Japan also found $F$. prausnitzii to be depleted in patients with inflammatory bowel disease. Sokol was particularly intrigued by the results from Japan. In East Asian populations the gene variants associated with inflammatory bowel disease differ from the gene variants in European populations. Yet the same bacterial species-F. prausnitzii—was reduced in the guts of those in whom the disease developed. This suggested that whereas different genetic vulnerabilities might underlie the disorder, the path to disease was similar: a loss of anti-inflammatory microbes from the gut. And although Sokol suspects change quickly over time in a single individual. The great majority of the microbes live in the gut, particularly the large intestine, which serves as an anaerobic digestion chamber. Scientists are still in the early stages of exploring the gut microbiome, but a burgeoning body of research suggests that the makeup of this complex microbial ecosystem is closely linked with our immune function. Some researchers now suspect that, aside from protecting us from infection, one of the immune system's jobs is to cultivate, or "farm," the friendly microbes that we rely on to keep us healthy. This "farming" goes both ways, though. Our resident microbes seem to control aspects of our immune function in a way that suggests they are farming us, too.

Independent researchers around the world have identified a select group of microbes that seem important for gut health and a balanced immune system. They belong to several clustered branches of the that other good bacteria besides F prausnitzii exist, this similarity hinted at a potential one-size-fits-all remedy for Crohn's and possibly other inflammatory disorders: restoration of peacekeeping microbes.

\section{MICROBIAL ECOSYSTEMS}

ONE OF THE QUESTIONS central to microbiome research is why people in modern society, who are relatively free of infectious diseases, a major cause of inflammation, are so prone to inflammatory, autoimmune and allergic diseases. Many now suspect that society-wide shifts in our microbial communities have contributed to our seemingly hyperreactive immune systems. Drivers of these changes might include antibiotics; sanitary practices that are aimed at limiting infectious disease but that also hinder the transmission of symbiotic microbes; and, of course, our high-sugar, high-fat modern diet. Our 
microbes eat what we eat, after all. Moreover, our particular surroundings may seed us with unique microbes, "localizing" our microbiota.

The tremendous microbial variation now evident among people has forced scientists to rethink how these communities work. Whereas a few years ago they imagined a core set of human-adapted microbes common to us all, they are now more likely to discuss core functions-specific jobs fulfilled by any number of microbes.

Faced with the many instances of a misbehaving immune system, it is tempting to imagine that rather than having developed a greater vulnerability to many diseases, we actually suffer from just one problem: a hyperreactive immune system. Maybe that tendency has been enabled, in part, by a decline or loss of key anti-inflammatory microbes and a weakening of their peacekeeping function.

\section{Antibiotics may deplete the bacteria that favorably calibrate the immune system, leaving it prone to overreaction.}

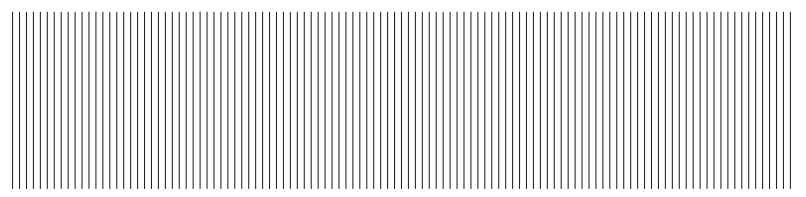

In ecosystem science, "keystone species" have an outsize role in shaping the greater ecosystem. Elephants, for example, help to maintain the African savanna by knocking down trees, thus benefiting all grazing animals. The concept may not apply perfectly to our inner microbial ecosystems - keystone species tend to be few in number, whereas peacekeeping microbes such as F. prausnitzii are quite numerous. Yet it provides a useful framework to think about those clostridial microbes.

They seem to occupy a particular ecological niche, sidled right up against the gut lining, which allows them to interface more closely with us, their hosts, than other members of the gut microbiota. They often specialize in fermenting dietary fiber that we cannot digest and produce by-products, or metabolites, that appear to be important for gut health. Some of the cells that line our colon derive nourishment directly from these metabolites, not from the bloodstream. And when no fiber comes down the hatch, the clostridial microbes and others can switch to sugars in the intestinal mucous layer - sugars we produce, apparently, to keep them happy. In fact, they seem to stimulate mucus production.

Kenya Honda, a microbiologist at Keio University in Tokyo, was among the first to uncover the critical role of clostridial microbes in maintaining a balanced immune system. To study how native microbes affect animals, scientists decades ago developed the germ-free mouse: an animal without any microbiota whatsoever. These rodents, delivered by cesarean section and raised in sterile plastic bubbles, can exist only in labs. Of the many oddities they present-including shrunken heart and lungs and abnormalities in the large intestine-Honda was particularly intrigued by their lack of cells that prevented immune overreaction, called regulatory $\mathrm{T}$ cells, or Tregs. Without these cells, the mice were unusually prone to inflammatory disease.

Honda wanted to know which of the many intestinal species might induce these suppressor cells. Soon after Sokol identified the anti-inflammatory effects of $F$. prausnitzii, Honda began whittling away at the gut microbiota of mice by treating them with narrowspectrum antibiotics. The animals' Tregs declined after a course of vancomycin. With their ability to restrain their immune reaction hobbled, the mice became highly susceptible to colitis, the rodent version of inflammatory bowel disease and allergic diarrhea. Honda found he could restore the Tregs and immune equilibrium of the mice just by reinstating 46 native clostridial strains.

Honda repeated the exercise with human-adapted microbes obtained from a healthy lab member. He extracted just 17 clostridial species this time that, in mice, could induce a full repertoire of Tregs and prevent inflammation. These human-adapted microbes specialized in nudging the immune system away from inflammatory disease. They came from branches of the clostridial group labeled clusters IV, XIVa and XVIII. F. prausnitzii belongs to cluster IV.

Vedanta Biosciences recently formed to try to turn Honda's 17-strain "clostridial cocktail" into a treatment for inflammatory disease. If the company's efforts are successful, it could signal the arrival of the next generation of probiotics-human-adapted microbes to treat immune-mediated disease - and all derived from one member of Honda's lab. As always, it is unclear if what works in lab mice will translate to humans. Sokol has his doubts. He recently identified a type of regulatory $\mathrm{T}$ cell that is unique to humans and that is deficient in people with inflammatory bowel disease. He questions if Honda's cocktail, which has been developed in mice, will activate these cells in people.

\section{TROUBLE WITH ANTIBIOTICS}

EVEN IF THE COCKTAIL falls short, Honda's meticulous demonstration of a link between antibiotics and vulnerability to inflammatory disease has raised a troubling question. A number of studies have found a small but significant correlation between the early-life use of antibiotics and the later development of inflammatory disorders, including asthma, inflammatory bowel disease and, more recently, colorectal cancer and childhood obesity. One explanation for this association might be that sickly people take more antibiotics. Antibiotics are not the cause, in other words, but the result of preexisting ill health.

Honda's studies suggest another explanation: antibiotics may deplete the very bacteria that favorably calibrate the immune system, leaving it prone to overreaction. Brett Finlay, a microbiologist at the University of British Columbia, has explored this possibility explicitly. Early-life vancomycin treatment of mice increased the animals' risk of asthma later, he found, in part by depleting those very same clostridial bacteria identified by Honda. The corresponding population of suppressor cells collapsed. And the animals became less able to restrain their immune responses when encountering allergens later.

These dynamics may also apply to other diseases. Earlier this year Cathryn Nagler, an immunologist at the University of Chicago, 
knocked out the clostridial bacteria with antibiotics and then fed the animals peanut protein. Without those microbes and their corresponding Tregs present, the protein leaked through the gut barrier into circulation, prompting the rodent version of a food allergy. She could prevent the sensitization just by introducing those clostridial bacteria.

One key difference between mice with and without the clostridial clusters was how many mucus-secreting cells they possessed. Animals that harbored the clostridial clusters had more. That may have farreaching consequences. Mucus, scientists are finding, contains compounds that repel certain microbes, maintaining a tiny distance between them and us. But it also carries food for other bacteriacomplex, fermentable sugars that resemble those found in breast milk. Lora Hooper, a microbiologist at the University of Texas Southwestern Medical Center in Dallas, calls this dual function the "carrot" and the "stick." Mucus serves both as an antimicrobial repellent and a growth medium for friendly bacteria.

This phenomenon matters for several reasons. As Nagler's experiments suggest, one way these clostridial clusters may promote gut health and a balanced immune system is by ensuring a healthy flow of mucus. Just as those elephants help to maintain the African savanna, these microbes may favorably shape the greater gut ecosystem by stimulating secretion of the sugars other friendly microbes graze on.

Conversely, scientists observe defects in the mucous layer in other disorders, particularly inflammatory bowel disease, where these clostridial bacteria are often depleted. The question has always been which comes first: defects in mucus secretion and the selection of an aberrant community of microbes or acquisition of an aberrant community of microbes that thins the mucous layer and increases vulnerability to disease? Both factors may work together.

In 2011 scientists at the University of Colorado Boulder sampled people with variants of a gene called NOD2 associated with inflammatory bowel disease. No one quite understands how these variants of the gene, which codes for a microbial sensor, increase the risk of disease. Study participants included people both with and without disease. Those suffering from inflammatory bowel disease had reduced counts of clostridial bacteria, the scientists found. But more surprising, people who did not have disease but who carried the predisposing NOD2 variants also had a relative depletion of clostridial clusters. Their microbial communities seemed positioned closer to a diseaselike state.

The study seems to highlight the role of genes in determining the composition of gut microbiota and the vulnerability to Crohn's. But epidemiological surveys complicate the picture. A number of studies over the years have linked having fewer sanitary amenities in childhood with a lower risk of inflammatory bowel disease in adulthood. And a 2014 study from Aarhus University in Denmark found that among northern Europeans, growing up on a farm with livestockanother microbially enriched environment - halved the risk of being stricken with inflammatory bowel disease in adulthood.

These patterns suggest that perhaps by seeding the gut microbiota early in life or by direct modification of the immune system the environment can affect our risk of inflammatory bowel disease despite the genes we carry. And they raise the question of what proactive steps those of us who do not live on farms can take to increase our chances of harboring a healthy mix of microbes.

\section{THE IMPORTANCE OF FIBER}

ONE OF THE MORE SURPRISING discoveries in recent years is how much the gut microbiota of people living in North America differs from those of people living in rural conditions in Africa and South America. The microbial mix in North America is geared to digesting protein, simple sugars and fats, whereas the mix in rural African and Amazonian environments is far more diverse and geared to fermenting plant fiber. Some think that our hunter-gatherer ancestors harbored even greater microbial diversity in their guts. If we accept the gut microbiota of people in rural Africa and South America as proxies for those that prevailed before the industrial revolution, then, says Justin L. Sonnenburg, a microbiologist at Stanford University, the observed differences suggest North Americans and other Westernized populations have veered into evolutionarily novel territory.

What troubles Sonnenburg about this shift is that the bacteria that seem most anti-inflammatory-including the clostridial clustersoften specialize in fermenting soluble fiber. Fermentation produces various metabolites, including butyrate, acetate and propionatesome of the substances that produce underarm odor. Various rodent studies suggest that these metabolites, called short-chain fatty acids, can induce Tregs and calibrate immune function in ways that, over a lifetime, may prevent inflammatory disease. Fermentation by-products may be one way our gut microbes communicate with our bodies. One takeaway is to "feed your Tregs more fiber," as University of Oxford immunologist Fiona Powrie put it last year in the journal Science.

Yet the seeming importance of these metabolites has others puzzled. Many bacteria produce these short-chain fatty acids, and yet only a few microbes seem potently anti-inflammatory. So although production of these metabolites may be a prerequisite for microbes that favorably tweak the immune system, says Sarkis Mazmanian, a microbiologist at the California Institute of Technology, it is insufficient to explain why some bacteria are more anti-inflammatory than others. Other characteristics, such as how close they live to the gut lining or the molecules they use to prod the host immune system, must also play a role, he says.

There is, however, an issue of sheer quantity. Some hunter-gatherers consumed up to 10 times as much soluble fiber as modern populations, and their bodies likely were flooded with far more fermentation by-products. Our fiber-poor modern diet may have weakened that signal, producing a state of "simmering hyperreactivity," Sonnenburg says, and predisposing us to the "plagues" of civilization. He calls this problem "starving our microbial self." We may not be adequately feeding some of the most important members of our microbiota.

Mouse experiments support the idea. Diets high in certain fats and sugars deplete anti-inflammatory bacteria, thin the mucous layer and foster systemic inflammation. Potentially dangerous opportunists bloom. In one intervention on human volunteers, University of California, San Francisco, microbiologist Peter Turnbaugh found that switching to a high-fat, high-protein diet spurred an expansion of bile-tolerant bacteria, one of which, Bilophila wadsworthia, has been 


\section{Your Microbes at Work: Fiber Fermenters Keep Us Healthy}

The gut houses trillions of microbes. They eat what you eat. Many specialize in fermenting the soluble fiber in legumes, grains, fruits and vegetables. Certain microbial species are adept at colonizing the mucous layer of the gut. Mucus contains antimicrobial substances that keep the microbiota at a slight distance. But it also contains sugars such as those found in breast milk. Some microbes, often the same ones that specialize in fermenting fiber, can use these sugars as sustenance when other food is not available. The by-products of fiber fermentation nourish cells lining the colon. Some by-products pass into the circulation and may calibrate our immune system in a way that prevents inflammatory disorders such as asthma and Crohn's disease.

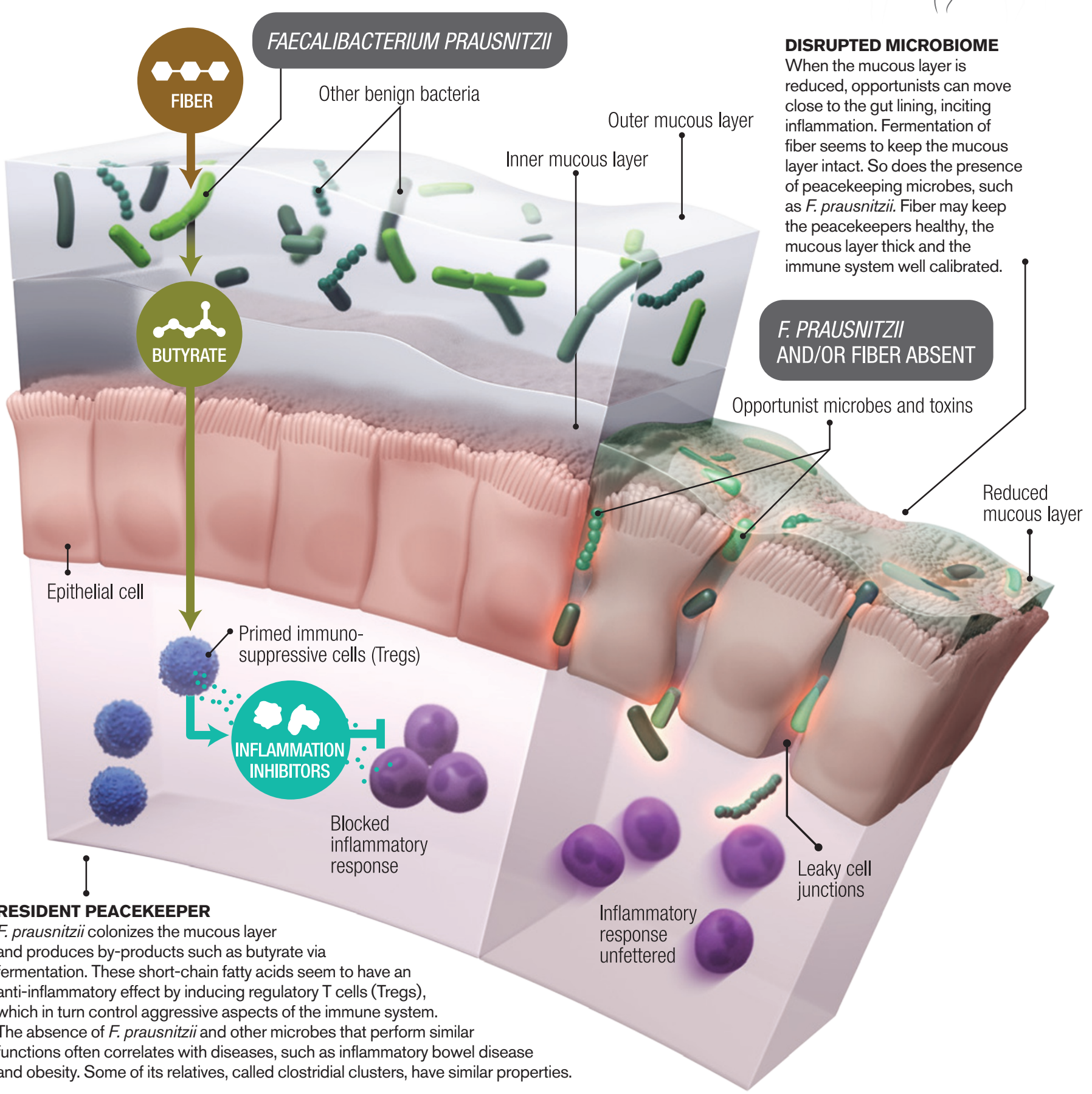


linked to inflammatory bowel disease. On the other hand, preventing this skewing of the microbial self does not seem that difficult. In rodents, adding fermentable fiber to a diet otherwise high in fat keeps the "good" microbes happy, the mucous layer healthy and the gut barrier intact, and it prevents systemic inflammation. Taken together, these studies suggest that it is not only what is in your food that matters for your health but also what is missing.

The human studies are even more intriguing. Mounting evidence suggests that the systemic inflammation observed in obesity does not just result from the accumulation of fat but contributes to it. Scientists at Catholic University of Louvain in Belgium recently showed that adding inulin, a fermentable fiber, to the diet of obese women increased counts of F prausnitzii and other clostridial bacteria and reduced that dangerous systemic inflammation. Weight loss was minor, but later analysis of this and two similar studies revealed that the intervention worked best on patients who, at the outset, already harbored clostridial clusters IV, IX and XIVa-some of the same clusters represented in Honda's cocktail. Those without the bacteria did not benefit, which suggests that once species disappear from the "microbial organ," the associated functions might also vanish. These individuals might not require ecosystem engineering so much as an ecosystem restoration.

That possibility has also been tested. Several years ago Max Nieuwdorp, a gastroenterologist at the Academic Medical Center in Amsterdam, transplanted microbes from lean donors to patients recently diagnosed with metabolic syndrome, a cluster of symptoms that often predicts type 2 diabetes. The recipients saw improvements in insulin sensitivity and an enrichment of their microbiota, including among those clostridial species. But six months after the transplant the patients had relapsed, metabolic improvements had faded and their microbes had reverted to their original states.

To Sonnenburg, this outcome suggests that the dance between human host and microbial community has considerable momentum. Removing the "diseased" ecosystem and installing a new one may not overcome the inertia. The gut immune system may simply mold the new community in the image of the old. That may explain why fecal transplants, which effectively vanquish $C$. difficile-associated diarrhea, have so far failed to treat inflammatory bowel disease. The former is caused by a single opportunist; the latter may be driven by an out-ofwhack ecosystem and our response to the microbial derangement.

To overcome the inertia, Sonnenburg foresees treating the host and the microbiota simultaneously. The idea has not been tested, but he imagines clearing out the microbiota, perhaps with antibiotics, followed by immunosuppressants to quiet the patient's immune system and allow healing. Only then might the new community of microbes stick and successfully recalibrate the immune system.

\section{EVOLUTION OF MOBILITY}

WHEN ANIMAL LIFE EXPLODED some 800 million years ago, microbes had already existed on Earth for maybe three billion years. A major innovation in animal evolution was the gut - a tube that takes nutrients in one end and expels waste from the other. It is even possible, argues Margaret McFall-Ngai, a microbiologist at the University of

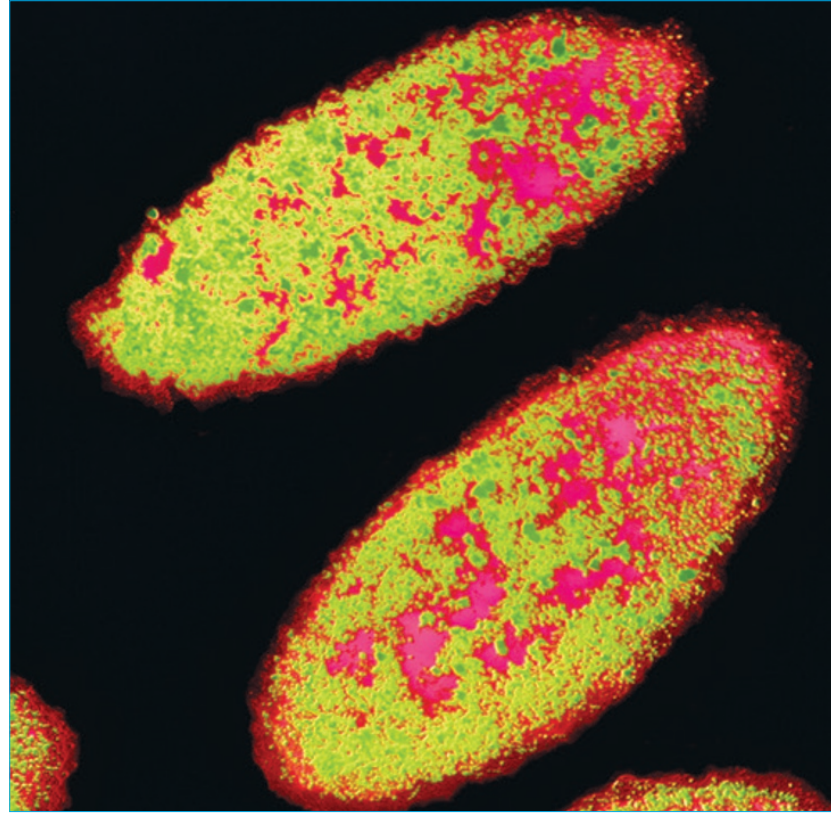

DANGEROUS OPPORTUNIST: Bilophila wadsworthia, a species of bacterium linked to inflammatory bowel disease, bloomed in the microbiota of human volunteers fed a high-fat, high-protein diet in a recent experiment.

Wisconsin-Madison, that microbes drove the evolution of the gut directly. Plants only succeeded in colonizing land when they had developed relationships with microbes that helped them extract vital nutrients from soil. Perhaps one evolutionary innovation of animals was to scoop up the microbial communities necessary for survival and to take them along for the ride, achieving mobility.

Mucus may be one way the human gut selects for these microbes. Only co-adapted bacteria, Sonnenburg thinks, can metabolize the complex sugars it contains. A cornerstone of this symbiosis may be the simple imperative of acquiring nutrients in a world of scarcity. We hunt and gather the goods; the microbes ferment what we cannot digest, taking a cut in the process and keeping pathogens at bay. Our immune systems quiet down when they receive signals, conveyed partly in microbial metabolites, indicating that the right microbes are in place.

The field of gut microbiome research has already moved from the idea of describing the core species to identifying the core ecological functions various microbes perform. Many potential species may fulfill any given role. Now another concept may be emerging, which might be called the keystone relationship. "The interaction between fiber and microbes that consume it," Sonnenburg says, "is the fundamental keystone interaction that everything else is built on in the gut." It may lie at the heart of the symbiotic pact between microbes and humans.

Moises Velasquez-Manoff is author of An Epidemic of Absence: A New Way of Understanding Allergies and Autoimmune Diseases. His work has appeared in the New York Times, Mother Jones and Nautilus. 